\title{
Identification of Prognostic Biomarkers and Immunotherapeutic Targets with CXC Chemokines in Glioblastoma Multiforme Using Integrated Bioinformatic Analysis
}

Hailing Liu

Maoming People's Hospital

Jinguang Zhu

Maoming People's Hospital

Guangwen Wang (D 929233133@qq.com )

https://orcid.org/0000-0002-9240-0356

Primary research

Keywords: CXC chemokines, glioblastoma multiforme, prognostic biomarker, therapeutic target, bioinformatic analysis

Posted Date: June 17th, 2020

DOI: https://doi.org/10.21203/rs.3.rs-35731/v1

License: (a) (1) This work is licensed under a Creative Commons Attribution 4.0 International License.

Read Full License 


\section{Abstract}

Background Glioblastoma multiforme (GBM) is the most malignant central nervous system tumour bearing a dismal prognosis. The study aimed to explore the potential biomarkers and therapeutic targets with CXC chemokines in GBM by integrated bioinformatics analysis.

Methods Differentially expressed CXC Chemokines were identified in GBM using GEPIA and UALCAN databases,and Kaplan-Meier analyses were performed by GEPIA subsequently. Protein -protein interaction (PPI) network was established in STRING database. Gene ontology (GO) and Kyoto Encyclopedia of Genes and Genomes (KEGG) pathways analysis were utilized to analyze differentially expressed CXC Chemokines and their similar genes gained from GEPIA. Then, we conducted transcription factors, kinase targets, and immune cells infiltration using TRRUST, LinkedOmics, and TIMER, respectively.

Results The mRNA expression levels of CXCL3/5/6/9/10/11/12/13/16 in GMB were significantly elevated compared to normal tissues. GBM patients with higher transcriptional levels of $C X C L 5 / 6$ were significantly associated with worse disease-free survival, while higher transcriptional levels of CXCL3/5/8 were significantly related to worse overall survival. The functions of CXC chemokines were enriched in Chemokine signaling pathway, Cytokine-cytokine receptor interaction, IL-17 signaling pathway, et.al. RELA and NFKB1 were key transcription factors of CXC chemokines. The kinase targets of CXC chemokine contained CDK1, CDK2, PRKCD, MAPK14, ATM, LCK, MTOR, and GRK3, which are involved in oncogenesis, migration, and survival. Moreover, we revealed significant correlations between the expression of CXC chemokines and the infiltration immune cells, especially for dendritic cells.

Conclusion The significant CXC chemokines and related pathways may provide a novel possibility for prognostic biomarkers and immunotherapeutic treatment in GMB.

Short title: CXC Chemokines with prognosis in GBM

\section{Background}

Glioblastoma multiforme (GBM) is the most malignant central nervous system tumour with dismal prognosis. Despite incremental advances in diagnostics and therapeutics including surgery, radiation, and chemotherapy, the 5-year survival rate of GBM patients is less than $15 \%{ }^{1}$. The cross-talk between cancer cells and immune cells is a crucial part in cancer growth, metastasis, and treatment effects, which is mediated in part by CXC chemokines in the tumor microenvironment ${ }^{2}$. Immunotherapy becomes an effective method of targeted therapy in tumors ${ }^{3}$. Recently, rapid progress in immunotherapy, such as immune- checkpoint inhibitors and cell-based vaccines have contributed to glioblastoma treatment ${ }^{4}$. However, it is still in the clinical trial, more molecular approaches contributing to clinically applicable biomarkers and therapeutic targets are needed. 
Chemokines are small chemotactic cytokines including CXC-chemokines, CCchemokines, Cchemokines, and CX3C-chemokines according to the position of their first two cysteine residues, which were expressed by tumour cells, immune cells, and stromal cells. It involved in leucocyte trafficking, angiogenesis, tumor growth, invasion, metastasis, and survival ${ }^{5}$. A complex chemokine network between immune-cell and leukocyte infiltration exists in turmor, which was related to tumour cell proliferation, migration, and survival ${ }^{6}$. Previous studies revealed that $\mathrm{CXC}$-chemokines were associated with GBM angiogenesis, proliferation, and migration ${ }^{7}$. However, identifying suitable CXC chemokines as prognostic biomarkers and therapeutic targets for GBM is elusive. Thus, a compelling attention to molecular basis and analysis is needed to extensively identify genomic abnormalities underlying GBM.

With the advent of next-generation sequencing technologies and the establishment of various public databases, comprehensive bioinformatics analysis of the expression of CXC chemokines in GBM has become possible. Our study using integrated bioinformatics analysis of the expression of CXC chemokines might provide valuable information for exploring potential new molecular biomarkers and therapeutic targets for GBM.

\section{Materials And Methods}

\section{UALCAN}

UALCAN (http://ualcan.path.uab.edu/analysis.html), is an interactive web-portal to perform analyses of The Cancer Genome Atlas (TCGA) level 3 RNA-seq and clinical data from 31 cancer types $^{8}$. In the study, we gained the data of CXC chemokines in GBM using the "TCGA Analysis" module of UALCAN and the "GBM" dataset, and then performed a differential mRNA expression analysis of GBM and normal tissues. The p-value was explored with Student's t-test and the cutoff was 0.05 .

\section{GEPIA}

GEPIA (http://gepia.cancer-pku.cn/index.html) is a web-based tool with extensively customize the visualization to deliver functionalities including differential expression analysis, profiling plotting, correlation analysis, patient survival analysis, similar gene detection and dimensionality reduction analysis based on TCGA and GTEx data ${ }^{9}$. In the study, we performed a differential mRNA expression analysis of tumor and normal tissues with the "GBM" dataset of GEPIA. Then we used GEPIA server to analyze the prognostic significance of differentially expressed CXC chemokines in GBM. The prognostic analysis was performed using a Kaplan-Meier curve. Data of "similar gene detection" were extracted to evaluate the interaction of CXC chemokines in GBM. Student's t-test was used to analyze the differentially expressed of CXC chemokines in GBM. The p-value cutoff was 0.05 .

\section{cBioPortal}

cBioPortal (www.cbioportal.org), a comprehensive web resource, provides mutation data, copy number alterations, microarray-based and RNA sequencing-based mRNA expression changes based on the TCGA- 
derived data ${ }^{10}$. We explored the genetic alteration of differentially expressed CXC chemokines with cBioPortal.

\section{STRING}

STRING (https://string-db.org/) is a web resource available online to construct the protein-protein interaction (PPI) network ${ }^{11}$. We explored gene coexpression with STRING. Moreover, we established a PPI network analysis of differentially expressed CXC chemokines to explore the interactions among genes in STRING database. A value of $\mathrm{P}<.05$ was seen to be statistically significant.

\section{R software}

Gene Ontology (GO) function and Kyoto Encyclopedia of Genes and Genomes (KEGG) pathways involving differentially expressed CXC chemokines and their similar genes were conducted using $\mathrm{R}$ software to analyze the function enrichment among the genes. A value of $P<.05$ was seen to be statistically significant.

\section{TRRUST}

TRRUST (https://www.grnpedia.org/trrust/) is a versatile database for the study of the transcriptional regulation, consisting of 8444 regulatory interactions for 800 transcription factors (TFs) in humans ${ }^{12}$. We gained the TF of CXC chemokines in GBM.

\section{LinkedOmics}

LinkedOmics (http://www.linkedomics.org/) is an open source portal available online, and provides a stage for biologists and clinicians to access, analyze and compare cancer multi-omics data within and across tumor types from The Cancer Genome Atlas (TCGA) project ${ }^{13}$. We used the "Linklnterpreter module" transforms identified associations into biological understanding through the pathway and network analysis of CXC chemokines in GBM.

\section{TIMER}

TIMER (https://cistrome.shinyapps.io/timer/) is a visual tool to comprehensively investigate molecular characterization of tumor-immune interactions ${ }^{14}$. In our study, we used "Gene module" to evaluate the correlation between CXC chemokines level and the infiltration of immune cells, including CD $4+T$ cells, CD8 + T cells, B cells, Macrophages, Neutrophils, and Dendritic cells, while we used "Survival module" to evaluate the correlation among clinical outcome and the infiltration of immune cells based on the GBM data from TCGA database.

\section{Results}

Aberrant expression of CXC chemokines in GBM vs normal tissue. 
Sixteen CXC chemokines except CXCL15 in GMB were conducted on the UALCAN database. We screened out nine increased mRNA expression levels for CXCL3, CXCL5, CXCL6, CXCL9, CXCL10, CXCL11, CXCL12, CXCL13, CXCL16 in GMB patients compared to normal tissues. The results are presented in Fig. 1.

Meanwhile, based on the database of GEPIA, the transcriptional levels of CXCL2, CXCL3, CXCL8, CXCL9, CXCL10, CXCL11, and CXCL16 in GBM tissues were significantly elevated compared to normal tissue (Fig. 2). All the differential expression gene were elevated. Combining the data from two databases of UALCAN and GEPIA, we gained eleven differentially expressed CXC chemokines in GBM totally (CXCL2, CXCL3, CXCL5, CXCL6, CXCL8, CXCL9, CXCL10, CXCL11, CXCL12, CXCL13, and CXCL16). CXC chemokines of CXCL1, CXCL4, CXCL7, and CXCL14 were excluded because of similar levels in GBM compared to normal tissue.

\section{The prognostic value of CXC chemokines in GBM}

We conducted Kaplan-Meier analysis of differentially expressed CXC chemokines and clinical outcome using GEPIA to assess the value of CXC chemokines in the prognosis of GMB. GBM patients with higher transcriptional levels of CXCL5 $(p=0.0086)$ and CXCL6 $(p=0.017)$ were significantly associated with worse disease-free survival (Fig. 3). Meanwhile, we found that GBM patients with higher transcriptional levels of CXCL3 $(p=0.021), \operatorname{CXCL5}(p=0.0046)$, and CXCL8 $(p=0.049)$ were significantly associated with worse overall survival (Fig. 4).

\section{The molecular characteristics, similar Gene, and PPI network analysis of CXC chemokines in patients with GBM}

We conducted an overall analysis of the molecular characteristics of differentially expressed CXC chemokines, including genetic alteration and gene coexpression. We gained the genetic alterations of differentially expressed CXC chemokines using cBioPortal, and found CXCL2, CXCL3, CXCL5, CXCL6, CXCL8, CXCL9, CXCL10, CXCL11, CXCL12, CXCL13, and CXCL16 were altered in 13\%, 6\%, 4\%, 6\%, 33\%, $7 \%, 21 \%, 8 \%, 6 \%, 5 \%$ and $0 \%$ in patients with GBM, respectively(Fig. $5 \mathrm{~A}$ ), which suggested high mRNA expression was the most common alteration. And then we explored the potential coexpression of the differentially expressed CXC chemokines with STRING (Fig. 5B). According to the coexpression scores, we revealed high correlation among CXCL9/10, CXCL9/10, CXCL8/2, and CXCL9/11, followed by CXCL3/8 and CXCL9/13. With the "similar gene detectioin" module of GEPIA, we identified additional genes with expression features similar to the differential expression $C X C$ chemokines. The top five similar genes of each CXC chemokines were selected, which including ZC3H12A,TACSTD2ロIGFL3ロRP11-

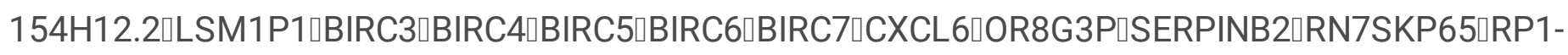

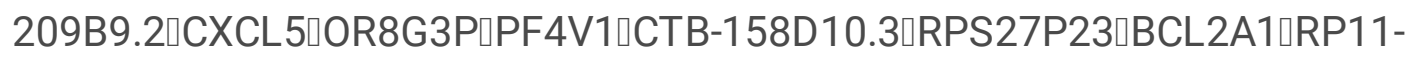

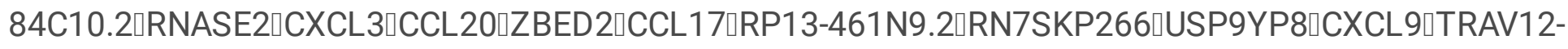

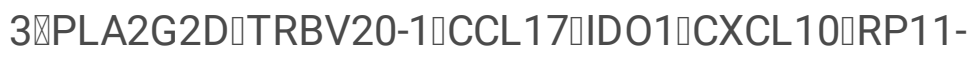

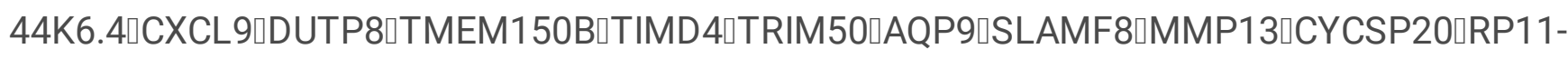

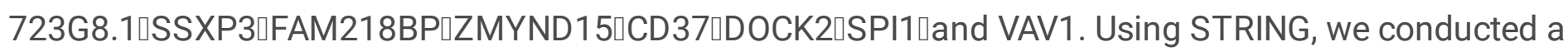
PPI network analysis of differentially expressed CXC chemokines, which contained 11 nodes and 55 
edges (Fig. 5C). The function of these differentially expressed CXC chemokines enriched in chemokine signaling pathway and inflammatory and immune response pathway.

\section{Functional enrichment analysis of the differentially expressed CXC chemokines and their similar genes in patients with GBM}

GO and KEGG enrichment analysis were used to explore the potential function of the differentially expressed CXC chemokines and their similar genes with R software (Fig. 6). GO analysis demonstrated that the differently expressed CXC chemokines and their similar genes were mostly enriched in BP (biological processes) category (response to chemokine, cellular response to chemokine, neutrophil migration, and et.al.) and MF (molecular function) category (chemokine activity, chemokine receptor binding, cytokine activity, and et.al.). CC category (cellular components) was absent. In addition, KEGG pathway analyses demonstrate that the top ten most significant pathways contained Chemokine signaling pathway, Viral protein interaction with cytokine and cytokine receptor, Cytokine-cytokine receptor interaction, IL-17 signaling pathway, Rheumatoid arthritis, TNF signaling pathway, NF-kappa B signaling pathway, Apoptosis - multiple species, Toll-like receptor signaling pathway, and Legionellosis.

\section{Transcription factor targets and kinase targets of CXC chemokines in patients with GBM}

To understand the context of CXC chemokines, we revealed possible transcription factor targets and kinase targets of the differentially expressed CXC chemokines with the resource of TRRUST and LinkedOmics respectively.

Query genes of CXCL2, CXCL5, CXCL8, CXCL10, CXCL12 included in TRRUST. Our data revealed two transcription factors (RELA and NFKB1) were the key regulators for gene targets of CXCL2, CXCL5, CXCL8, CXCL10, and CXCL12 (Table 1). We selected the top two kinase targets of CXC chemokines except CXC8/16 with LinkedOmics database. Kinase-target network of CXCL2 is related to PRKCD and MAPK14, while network of CXCL3 and CXCL9 is correlated to CDK2 and ATM. CDK2 and CDK1 were suggested as targets for CXCL5, CXCL6, CXCL11, and CXCL13 kinase-target network. CXCL10 kinasetarget network were generally associated with LCK and MTOR, and CXCL12 is related to PRKCD and GRK3 (Table 2).

Table 1

Results of candidate key regulators from TRRUST.

\begin{tabular}{|lllll|}
\hline Key TF & description & Regulated targets & $\begin{array}{l}\text { P } \\
\text { value }\end{array}$ & FDR \\
\hline RELA & $\begin{array}{l}\text { V-rel reticuloendotheliosis viral oncogene homolog } \\
\text { A (avian) }\end{array}$ & CXCL2/5/8/10/12 & $\begin{array}{l}4.26 \mathrm{e}- \\
07\end{array}$ & $\begin{array}{l}4.4 \mathrm{e}- \\
07\end{array}$ \\
\hline NFKB1 & $\begin{array}{l}\text { nuclear factor of kappa light polypeptide gene } \\
\text { enhancer in B-cells 1 }\end{array}$ & CXCL2/5/8/10/12 & $\begin{array}{l}4.4 \mathrm{e}- \\
07\end{array}$ & $\begin{array}{l}4.4 \mathrm{e}- \\
07\end{array}$ \\
\hline TF: transcription factor & & & \\
\hline
\end{tabular}


Table 2

Enriched kinase targets of CXC chemokines from LinkerOmics.

\begin{tabular}{|c|c|c|c|c|}
\hline $\begin{array}{l}\text { CXC } \\
\text { chemokines }\end{array}$ & $\begin{array}{l}\text { Enriched kinase } \\
\text { target }\end{array}$ & Description & $\begin{array}{l}\text { Leading Edge } \\
\text { Number }\end{array}$ & $\begin{array}{l}\mathrm{P} \\
\text { Value }\end{array}$ \\
\hline \multirow[t]{2}{*}{ CXCL2 } & Kinase_PRKCD & protein kinase $\mathrm{C}$ delta & 31 & 0 \\
\hline & Kinase_MAPK14 & mitogen-activated protein kinase 14 & 27 & 0.004 \\
\hline \multirow[t]{2}{*}{ CXCL3 } & Kinase_CDK2 & cyclin dependent kinase 2 & 75 & 0 \\
\hline & Kinase_ATM & ATM serine/threonine kinase & 60 & 0 \\
\hline \multirow[t]{2}{*}{ CXCL5 } & Kinase_CDK2 & cyclin dependent kinase 2 & 118 & 0 \\
\hline & Kinase_CDK1 & cyclin dependent kinase 1 & 104 & 0 \\
\hline \multirow[t]{2}{*}{ CXCL6 } & Kinase_CDK1 & cyclin dependent kinase 1 & 123 & 0 \\
\hline & Kinase_CDK2 & cyclin dependent kinase 2 & 107 & 0 \\
\hline \multirow[t]{2}{*}{ CXCL9 } & Kinase_CDK2 & cyclin dependent kinase 2 & 89 & 0 \\
\hline & Kinase_ATM & ATM serine/threonine kinase & 48 & 0 \\
\hline \multirow[t]{2}{*}{ CXCL10 } & Kinase_LCK & $\begin{array}{l}\text { LCK proto-oncogene, Src family } \\
\text { tyrosine kinase }\end{array}$ & 19 & 0 \\
\hline & Kinase_MTOR & mechanistic target of rapamycin & 19 & 0 \\
\hline \multirow[t]{2}{*}{ CXCL11 } & Kinase_CDK2 & cyclin dependent kinase 2 & 83 & 0 \\
\hline & Kinase_CDK1 & cyclin dependent kinase 1 & 79 & 0 \\
\hline \multirow[t]{2}{*}{ CXCL12 } & Kinase_PRKCD & protein kinase $\mathrm{C}$ delta & 28 & 0 \\
\hline & Kinase_GRK3 & G protein-coupled receptor kinase 3 & 24 & 0 \\
\hline \multirow[t]{2}{*}{ CXCL13 } & Kinase_CDK1 & cyclin dependent kinase 1 & 104 & 0 \\
\hline & Kinase_CDK2 & cyclin dependent kinase 2 & 99 & 0 \\
\hline
\end{tabular}

\section{Immune cell infiltration of CXC chemokines in patients with GBM}

To systematical analysis of immune infiltrates across GBM, we use "Gene module" module of TIMER to explore the correlation between differentially expressed CXC chemokine (not including CXCL8) and abundance of immune infiltrates (B cells, CD4 + T cells, CD8 + T cells, Neutrophils, Macrophages, and Dendritic cells). CXCL2 expression was negatively associated with the infiltration of CD8 $+\mathrm{T}$ cells (Cor = $-0.148, p=0.002$ ), and positively associated with the infiltration of neutrophils (Cor $=0.203, p=2.76 \mathrm{E}-05$ ) and dendritic cell (Cor $=0.400, p=1.67 \mathrm{E}-17$ ) (Fig. 7A). CXCL3 expression was negatively associated with the infiltration of $B$ cells (Cor $=-0.102, p=0.036$ ) and macrophage (Cor $=-0.130, p=0.007$ ), and positively associated with the infiltration of neutrophils (Cor $=0.122, p=0.012)$ and dendritic cell $(\operatorname{Cor}=0.389, p=$ 
1.31E-16) (Fig. 7B). CXCL5 expression was negatively associated with the infiltration of B cells (Cor = $-0.109, p=0.024)$ and $C D 8+T$ Cell (Cor $=-0.154, p=0.001)$, and positively associated with the infiltration of dendritic cell (Cor $=0.320, p=2.04 \mathrm{E}-11)$ (Fig. 7C). CXCL6 expression was negatively associated with the infiltration of $B$ cells (Cor $=-0.149, p=0.002$ ) and CD $8+T$ Cell (Cor $=-0.099, p=0.042$ ), and positively associated with the infiltration of dendritic cell (Cor $=0.332, p=2.82 \mathrm{E}-12)$ (Fig. 7D). CXCL9 expression was negatively associated with the infiltration of CD8 + T Cell (Cor $=-0.220, p=5.50 E-06)$ and CD $4+T$ Cell (Cor $=-0.151, p=0.0018)$, and positively associated with the infiltration of $B$ cells (Cor $=0.302, p=$ 2.84E-10) dendritic cell (Cor $=0.175, p=3.15 E-4)$ (Fig. 7E). CXCL10 expression was negatively associated with the infiltration of CD8 $+\mathrm{T}$ Cell (Cor $=-0.172, p=4.05 \mathrm{E}-04)$ and CD4 $+\mathrm{T}$ Cell $($ Cor $=-0.131, p=0.007)$, and positively associated with the infiltration of $B$ cells ( $\mathrm{Cor}=0.309, p=1.03 \mathrm{E}-10)$, neutrophil (Cor $=0.123$, $p=0.011$ ), and dendritic cell (Cor $=0.216, p=7.60 \mathrm{E}-06)$ (Fig. 7F). CXCL11 expression was negatively associated with the infiltration of CD4 $+T$ Cell (Cor $=-0.120, p=0.013$ ), and positively associated with the infiltration of $B$ cells (Cor $=0.302, p=2.86 E-10)$ and dendritic cell (Cor $=0.109, p=0.025)$ (Fig. 7G). CXCL12 expression was negatively associated with the infiltration of CD8 + T Cell (Cor $=-0.185, p=1.38 \mathrm{E}-$ 4 ), and positively associated with the infiltration of neutrophil (Cor $=0.111, p=0.023$ ) and dendritic cell (Cor $=0.287, p=2.18 \mathrm{E}-09$ ) (Fig. 7H). CXCL13 expression was negatively associated with the infiltration of CD8 + T Cell (Cor $=-0.236, p=1.01 E-06)$ and CD $4+T$ Cell (Cor $=-0.127, p=0.009$ ), and positively associated with dendritic cell (Cor $=0.101, p=0.037)$ (Fig. 7I). CXCL16 expression was negatively associated with the infiltration of CD8 $+\mathrm{T}$ Cell (Cor $=-0.281, p=0.001)$, and positively associated with CD4 + T Cell (Cor $=0.349, p=3.92 \mathrm{E}-05)$, macrophage (Cor $=0.186, p=0.032)$, and dendritic cell (Cor $=$ $0.340, p=6.43 \mathrm{E}-05$ ) (Fig. $7 \mathrm{~J}$ ). Our data revealed all the differentially expressed CXC chemokines are positively with infiltration of dendritic cell. In addition, we evaluated correlation of prognosis and immune cell infiltration in GBM using the Cox proportional hazard model. We found lower dendritic cell $(p=0.025)$ were significantly associated with longer cumulative survival of GMB (Fig. 7K). Previous study reported the autologous dendritic cell vaccine may extend survival in GBM patients ${ }^{15}$, suggesting CXC chemokines might be the potential therapeutic targets with modulating infiltration of dendritic cell.

\section{Discussion}

Chemokines involved in leucocyte trafficking, angiogenesis, tumor growth, invasion, metastasis, and survival ${ }^{5}$. Previous studies ${ }^{3,16}$ have reported a correlation among CXC chemokines, the tumor microenvironment, and cancer immunotherapy, suggesting that CXC chemokines may modulate tumor progression and immunotherapeutic effect. However, integrative bioinformatics analysis of CXC chemokines to evaluate the progress in GMB has not been well reported.

The eleven differentially expressed genes (CXCL2, CXCL3, CXCL5, CXCL6, CXCL8, CXCL9, CXCL10, CXCL11, CXCL12, CXCL13, and CXCL16) were gained in GBM compared to normal tissue using website of UALCAN and GEPIA, all of which are upregulation. In addition, we found that GBM patients with high transcriptional levels of CXCL5 and CXCL6 were significantly associated with worse disease-free survival. Moreover, GBM patients with high transcriptional levels of CXCL3, CXCL5, and CXCL8 were significantly 
associated with worse overall survival. The data suggested crucial relevance may exist between differentially expressed CXC chemokines and GBM, especially the outcome of GBM. Many studies reported that CXCL16 mRNA was particularly high in GBM. ${ }^{17}$ Expression of CXCL10 have an important role in the proliferation of glioma cells, which associated with increased malignancy grade ${ }^{18}$. Moreover, CXCL12 showed tumour-promoting properties on cultured glioma cells. ${ }^{19}$ Nevertheless, prognostic value as biomarker of CXC chemokines in GBM is absent.

To overall understanding molecular characteristics of CXC chemokines, we gained genetic alteration and coexpression. High mRNA expression was the most common mutation alteration of CXC chemokines in GBM, which was consistent with the upregulation of differential expressed CXC chemokines ${ }^{7}$, demonstrated CXC chemokines might correlate with the genesis and development of GBM. The result of CXC chemokines coexpression suggested CXC chemokines aced as an orchestra in GBM.

We then explored the function of differentially expressed CXC chemokines and their similar genes using GO and KEGG pathway enrichment analysis. We found that the functions of the related genes are mainly correlated to the cytokine and cytokine receptor, Cytokine-cytokine receptor interaction, IL-17 signaling pathway, TNF signaling pathway, and NF-kappa B signaling. Previous studies have demonstrated that chemokine signaling pathways and cytokine-cytokine receptor interactions play critical roles in cancer cell proliferation, angiogenesis, metastasis, and survival of various cancers ${ }^{7,18,20}$. IL-17 signaling is associated with immunopathology and cancer progression ${ }^{21}$. TNF signaling pathway and NF-kappa B signaling pathway play a significant role in cell proliferation and oncogenesis ${ }^{22}$. The data suggested that the CXC chemokines might be the new therapeutic targets responding to the pathway for GBM.

We found that two transcription factors (RELA and NFKB1) were associated with the regulation of CXC chemokines. RELA is the downstream transcriptional activators of the canonical NF-KB pathway, and regulate the activity and function of NF-KB including cell differentiation, apoptosis, and tumorigenesis, with various post-translational modifications, especially phosphorylation. A study demonstrated that RELA is a mediator of oncogene of pancreatic ductal adenocarcinoma, and the beneficial effects of RELA were mediated by increased expression of CXCL1 and CXCR2 ${ }^{23}$. NFKB1 as a suppressor of the NF-KB response contributed to tumorigenesis in many types of cancer, including $\mathrm{GBM}^{24}$. The cumulative data demonstrated CXC chemokine and NF-KB pathway may become potentially molecular targets in the clinical treatment of GBM. The probable targets of the differentially expressed CXC chemokines suggested CDK1, CDK2, PRKCD, MAPK14, ATM, LCK, MTOR, and GRK3. CDK1 is a critical modulator in the initiation and transition process from the $\mathrm{G} 2$ phase into mitosis of the cell cycle and has been proposed as a tumor specific anti-cancer target ${ }^{25}$. CDK2 is mainly associated with tumor growth in multiple cancer types, and CDK2 inhibition may provide a therapeutic benefit against certain tumors ${ }^{26}$. PRKCD is known to be an important regulator of apoptosis ${ }^{27}$. ATM was related to DNA damage response, and utilization of ATM inhibitors may provide a novel anti-cancer treatment strategy. LCK is expressed in the brain and in tumor cells, where it regulates cellular functions like proliferation, survival, and memory ${ }^{28}$. mTOR is frequently activated in cancer, which is associated with controls cell growth and 
metabolism ${ }^{29}$. GRK3 dysregulation may play an important part in the metastasis of tumor. Summary, the kinases are involved in cell cycle modulation, DNA damage, apoptosis, metastasis, and survival. Therefore, we inferred differentially expressed CXC chemokines may modulate tumor cell migration, invasion, and apoptosis in GBM by regulating these kinases.

Tumour microenvironment consisted of chemokines, chemokine receptors, and different immune cell subsets, which had distinct effects on tumour progression and therapeutic outcomes ${ }^{3}$. Chemokines can recruit specific immune cells into the tumour microenvironment to form tumour immunity and therapeutic responses. In our study, the differently expressed CXC-chemokines were accompanied by various immune cell infiltration, especially all of which were positively associated with dendritic cell. Moreover, we found lower dendritic cell were significantly associated with longer cumulative survival of GMB. Previous evidences ${ }^{15}, 30$ revealed the dendritic cell were associated with progress and acted as immunity therapeutic targets in GBM in phase III clinical trial. Collectedly, CXC-chemokines might be a potentially effective approach for predicting clinical outcome and target treating in GBM.

\section{Conclusion}

In a word, the significant chemokines and pathways may open up brand-new possibilities for prognostic biomarkers and immunotherapeutic treatment of GMB; however, further researches are still required for untangling the mechanism of occurrence and development of GBM.

\section{Abbreviations}

GBM: Glioblastoma multiforme; PPI: Protein -protein interaction; GO: Gene ontology; KEGG: Kyoto Encyclopedia of Genes and Genomes; TCGA: The Cancer Genome Atlas; TFs: transcription factors; BP: biological processes; MF: molecular function; CC: category cellular components.

\section{Declarations}

\section{Ethics approval and consent to participate}

The study was not a clinical trial. All data generated or analysed during this study are included in the public database.

\section{Funding}

This work was supported by High-level Hospital Construction Research Project of Maoming People's Hospital.

\section{Acknowledgements}

Not applicable. 


\section{Competing interests}

The authors declare that they have no competing interests.

\section{Availability of data and materials}

The datasets used and/or analyzed during the current study are available from the corresponding author on reasonable request.

\section{Consent for publication}

Not applicable.

Authors' contributions: WGW designed the study, LHL contributed to drafting the manuscript, ZJG read and approved the final manuscript.

\section{References}

1. Wirsching HG, Galanis E, Weller M. Glioblastoma Handb Clin Neurol. 2016;134:381-97.

2. Balkwill F. Cancer and the chemokine network. Nat Rev Cancer. 2004;4:540-50.

3. Nagarsheth N, Wicha MS, Zou W. Chemokines in the cancer microenvironment and their relevance in cancer immunotherapy. Nat Rev Immunol. 2017;17:559-72.

4. Wilcox JA, Ramakrishna R, Magge R. Immunotherapy in Glioblastoma. World Neurosurg. 2018;116:518-28.

5. Vandercappellen J, Van Damme J, Struyf S. The role of CXC chemokines and their receptors in cancer. Cancer Lett. 2008;267:226-44.

6. Zeng Q, Sun S, Li Y, Li X, Li Z, Liang H. Identification of Therapeutic Targets and Prognostic Biomarkers Among CXC Chemokines in the Renal Cell Carcinoma Microenvironment. Front Oncol. 2019;9:1555.

7. Christofides A, Kosmopoulos M, Piperi C. Pathophysiological mechanisms regulated by cytokines in gliomas. Cytokine. 2015;71:377-84.

8. Chandrashekar DS, Bashel B, Balasubramanya SAH, et al. UALCAN: A Portal for Facilitating Tumor Subgroup Gene Expression and Survival Analyses. Neoplasia. 2017;19:649-58.

9. Tang Z, Li C, Kang B, Gao G, Li C, Zhang Z. GEPIA: a web server for cancer and normal gene expression profiling and interactive analyses. Nucleic Acids Res. 2017;45:W98-102.

10. Gao J, Aksoy BA, Dogrusoz U, et al. Integrative analysis of complex cancer genomics and clinical profiles using the cBioPortal. Sci Signal. 2013;6:pl1.

11. Szklarczyk D, Gable AL, Lyon D, et al. STRING v11: protein-protein association networks with increased coverage, supporting functional discovery in genome-wide experimental datasets. Nucleic Acids Res. 2019;47:D607-13. 
12. Han H, Cho JW, Lee S, et al. TRRUST v2: an expanded reference database of human and mouse transcriptional regulatory interactions. Nucleic Acids Res. 2018;46:D380-6.

13. Vasaikar SV, Straub P, Wang J, Zhang B. LinkedOmics: analyzing multi-omics data within and across 32 cancer types. Nucleic Acids Res. 2018;46:D956-63.

14. Li T, Fan J, Wang B, et al. TIMER: A Web Server for Comprehensive Analysis of Tumor-Infiltrating Immune Cells. Cancer Res. 2017;77:e108-10.

15. Reardon DA, Mitchell DA. The development of dendritic cell vaccine-based immunotherapies for glioblastoma. Semin Immunopathol. 2017;39:225-39.

16. Gutmann DH. The Sociobiology of Brain Tumors. Adv Exp Med Biol. 2020;1225:115-25.

17. Ludwig A, Schulte A, Schnack $C$, et al. Enhanced expression and shedding of the transmembrane chemokine CXCL16 by reactive astrocytes and glioma cells. J Neurochem. 2005;93:1293-303.

18. Maru SV, Holloway KA, Flynn G, et al. Chemokine production and chemokine receptor expression by human glioma cells: role of CXCL10 in tumour cell proliferation. J Neuroimmunol. 2008;199:35-45.

19. Yi L, Zhou X, Li T, et al. Notch1 signaling pathway promotes invasion, self-renewal and growth of glioma initiating cells via modulating chemokine system CXCL12/CXCR4. J Exp Clin Cancer Res. 2019;38:339.

20. Domanska UM, Kruizinga RC, den Dunnen WF, Timmer-Bosscha H, de Vries EG, Walenkamp AM. The chemokine network, a newly discovered target in high grade gliomas. Crit Rev Oncol Hematol. 2011;79:154-63.

21. Amatya N, Garg AV, Gaffen SL. IL-17 Signaling: The Yin and the Yang. Trends Immunol. 2017;38:310-22.

22. Concetti J, Wilson CL. NFKB1 and Cancer: Friend or Foe? Cells 2018;7.

23. Kabacaoglu D, Ruess DA, Ai J, Algul H. NF-kappaB/Rel Transcription Factors in Pancreatic Cancer: Focusing on RelA, c-Rel, and RelB. Cancers (Basel) 2019;11.

24. Wang F, Jiang H, Wang S, Chen B. Dual Functional MicroRNA-186-5p Targets both FGF2 and RelA to Suppress Tumorigenesis of Glioblastoma Multiforme. Cell Mol Neurobiol. 2017;37:1433-42.

25. Wang Q, Su L, Liu N, Zhang L, Xu W, Fang H. Cyclin dependent kinase 1 inhibitors: a review of recent progress. Curr Med Chem. 2011;18:2025-43.

26. Tadesse S, Caldon EC, Tilley W, Wang S. Cyclin-Dependent Kinase 2 Inhibitors in Cancer Therapy: An Update. J Med Chem. 2019;62:4233-51.

27. Holmgren C, Cornmark L, Lonne GK, Masoumi KC, Larsson C. Molecular characterization of protein kinase C delta (PKCdelta)-Smac interactions. BMC Biochem. 2016;17:11.

28. Bommhardt U, Schraven B, Simeoni L. Beyond TCR, Signaling. Emerging Functions of Lck in Cancer and Immunotherapy. Int J Mol Sci 2019;20.

29. Mossmann D, Park S, Hall MN. mTOR signalling and cellular metabolism are mutual determinants in cancer. Nat Rev Cancer. 2018;18:744-57. 
30. Lim M, Xia Y, Bettegowda C, Weller M. Current state of immunotherapy for glioblastoma. Nat Rev Clin Oncol. 2018;15:422-42.

\section{Figures}
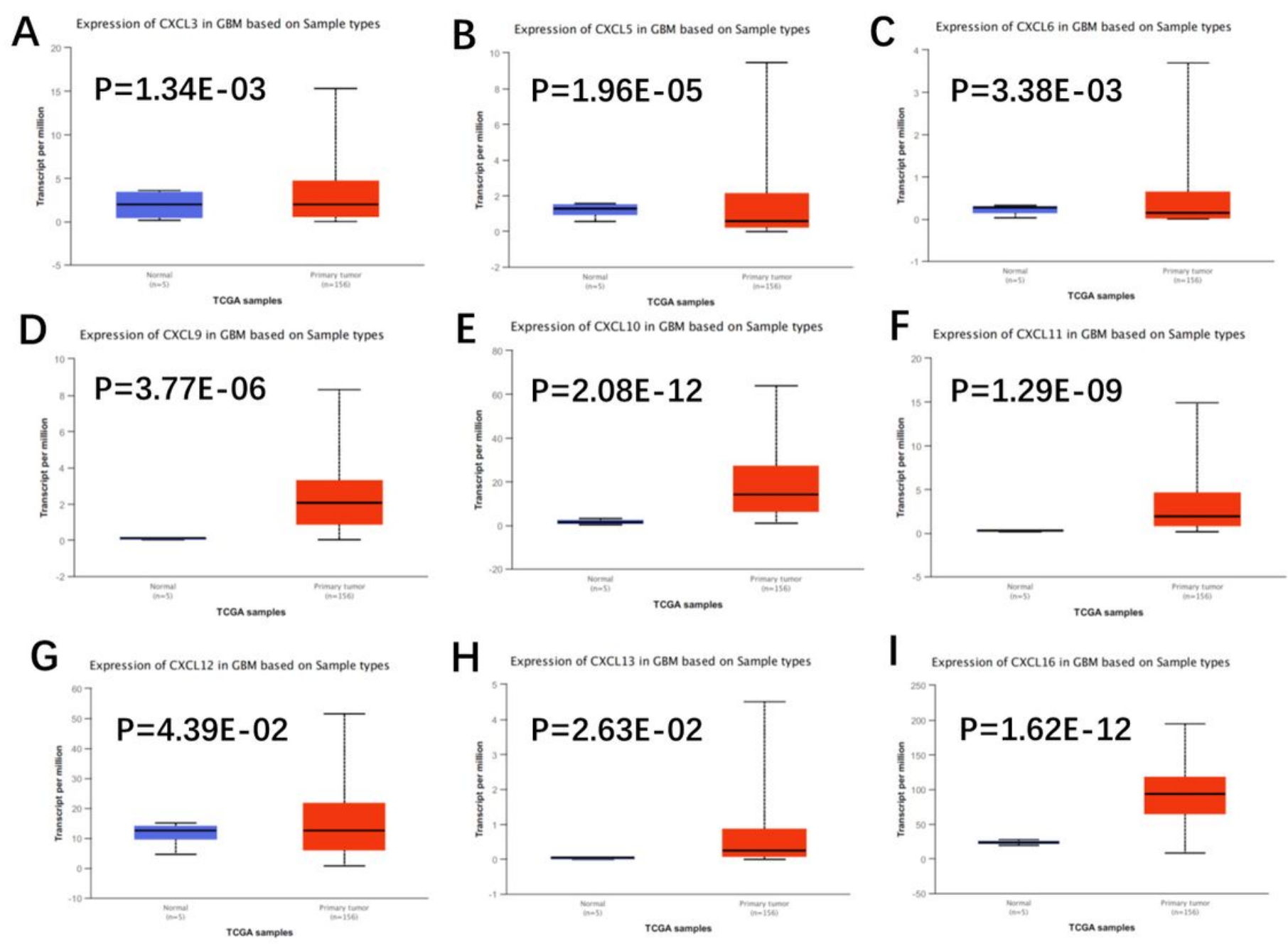

Figure 1

Aberrant expression of CXC chemokines in GBM from UALCAN database. The transcriptional levels of CXCL3/5/6/9/10 /11/12/13/16 in GBM tissues were significantly elevated compared to normal tissue. 

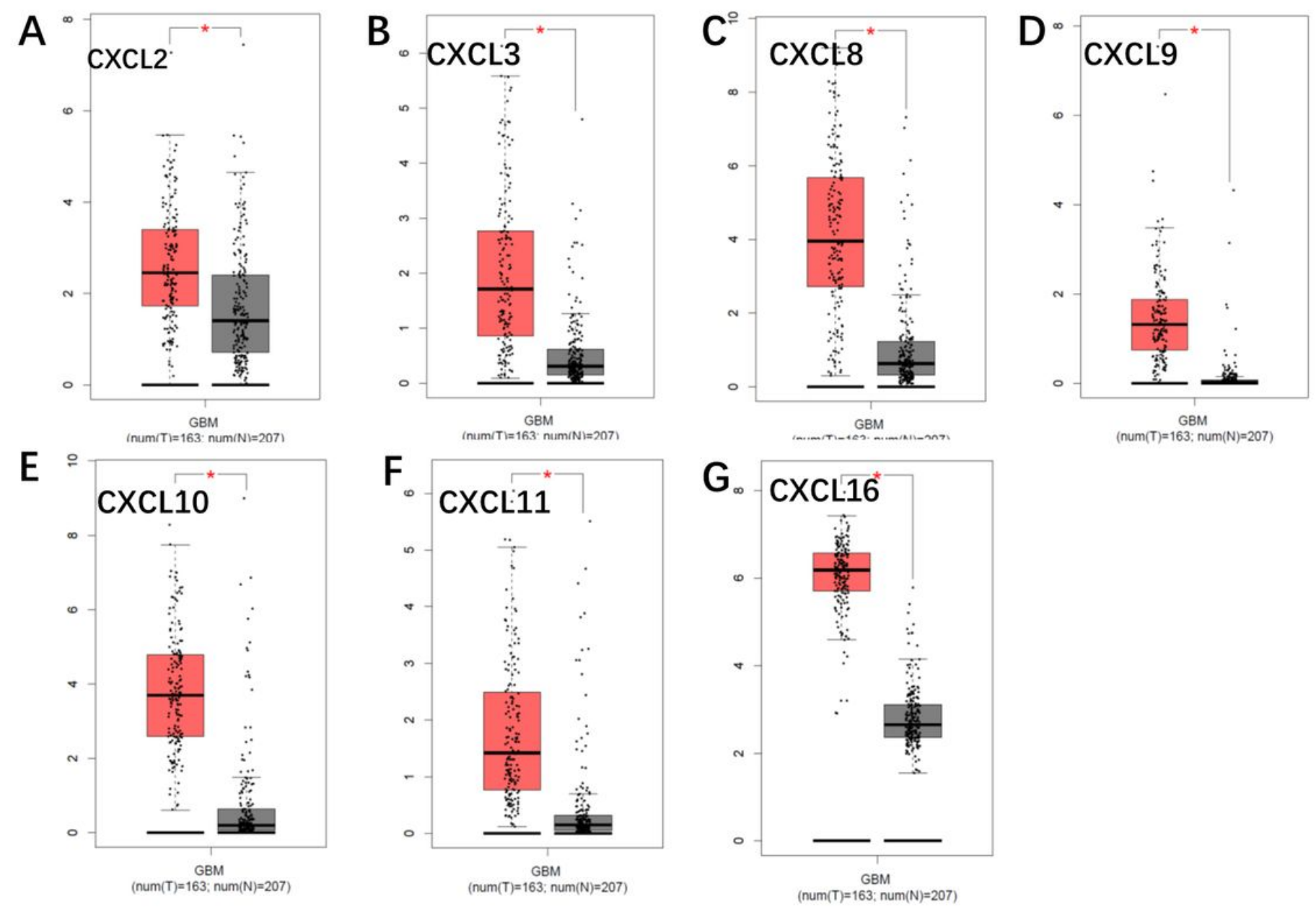

Figure 2

The transcriptional of CXC chemokines in GBM from GEPIA database. The transcriptional levels of CXCL2/3/8/9/10/11/16 were significantly increased in GBM vs normal tissue. 

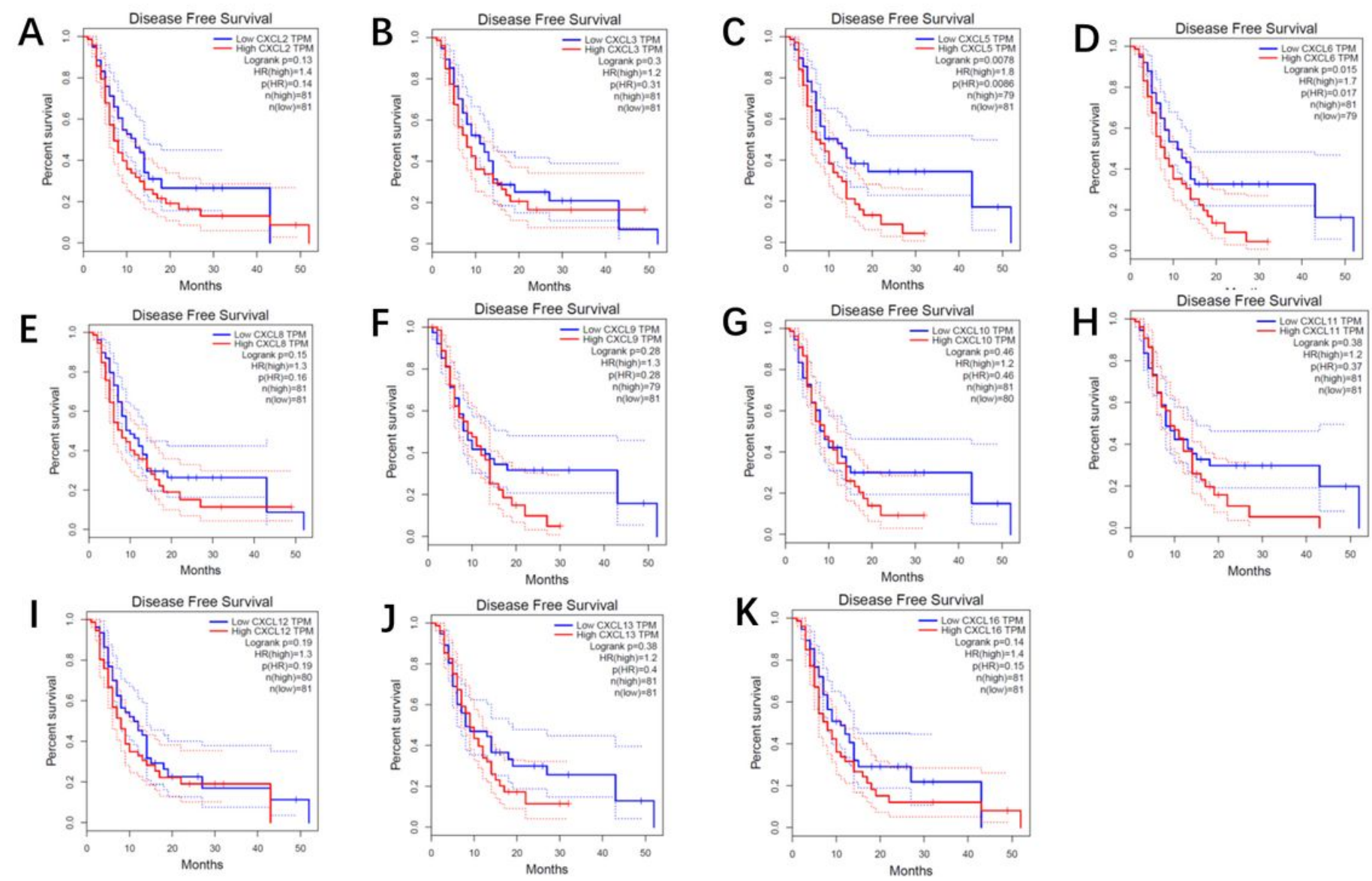

\section{Figure 3}

Prognostic values of differentially expressed CXC chemokines for disease free survival in GBM patients using GEPIA. 

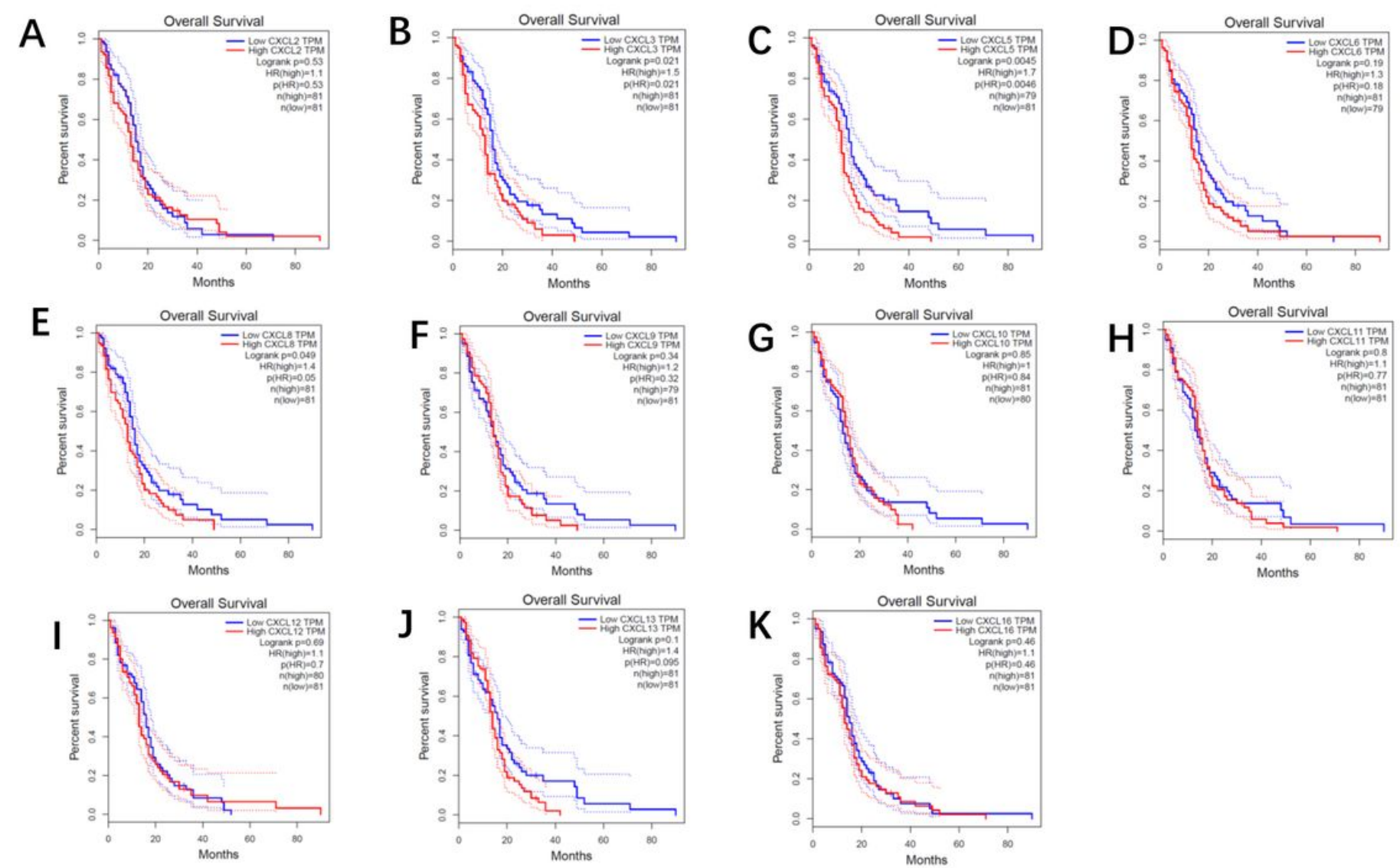

Figure 4

Prognostic values of differentially expressed CXC chemokines for overall survival in GBM patients using GEPIA. 


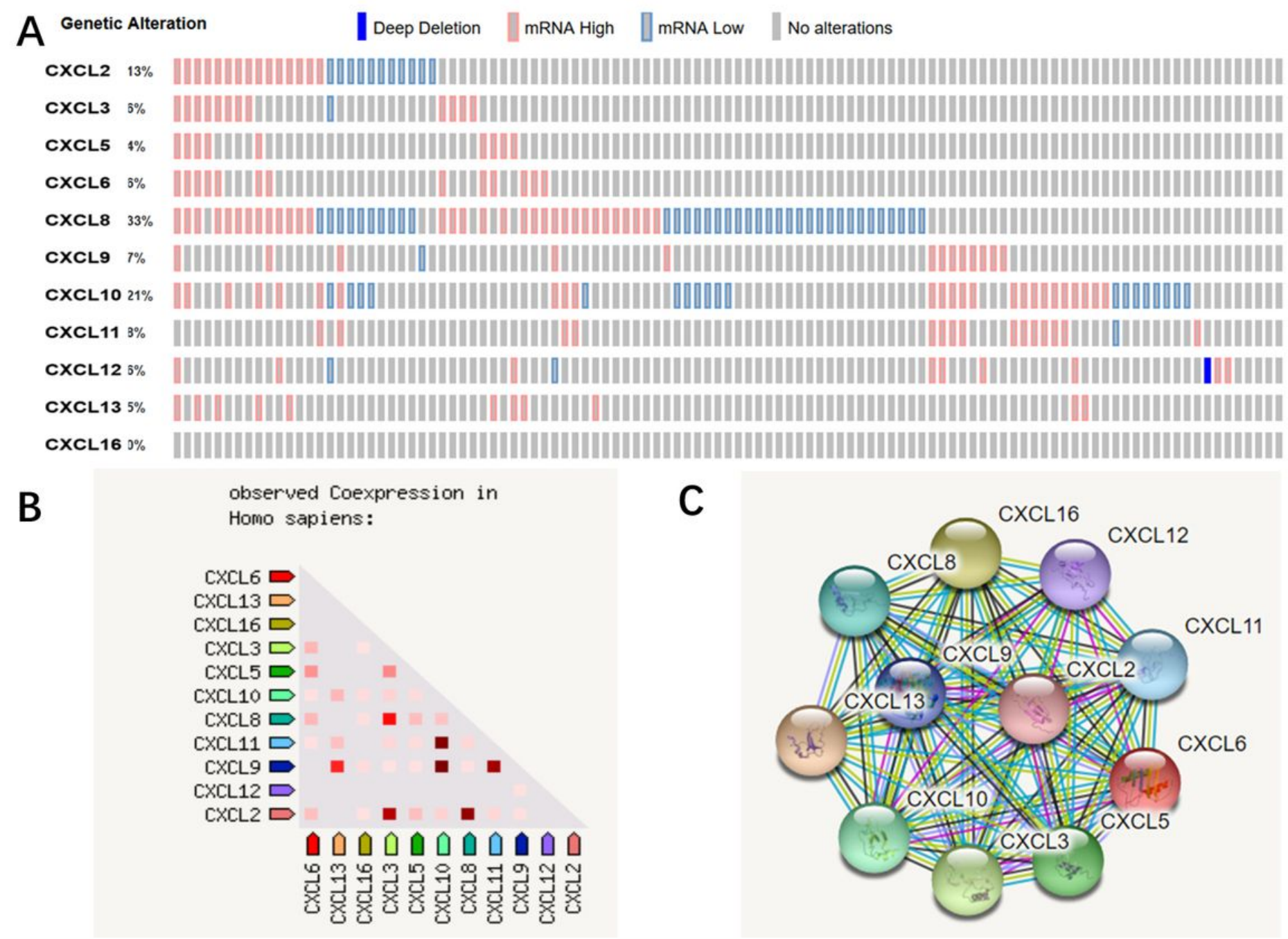

Figure 5

The molecular characteristics and PPI network analysis of CXC Chemokines in Patients with GBM. A. Genetic alteration of differentially expression CXC chemokine in GBM. B. Gene coexpression of differentially expression CXC chemokine in GBM with STRING. C. Protein-protein interaction network of differentially expressed CXC chemokines with STRING. 
A

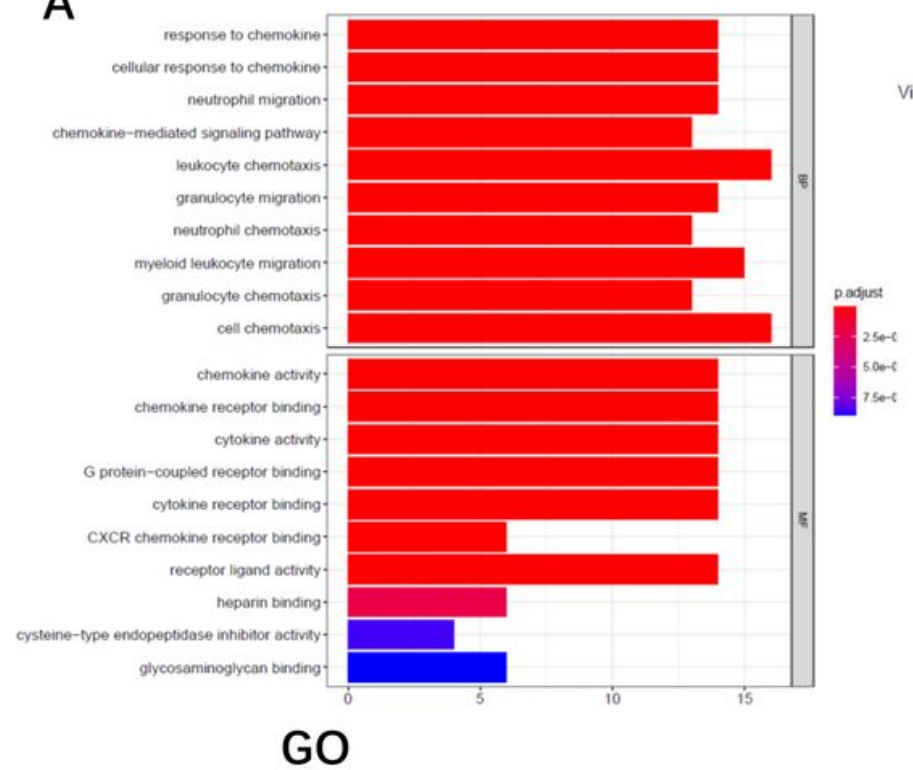

B

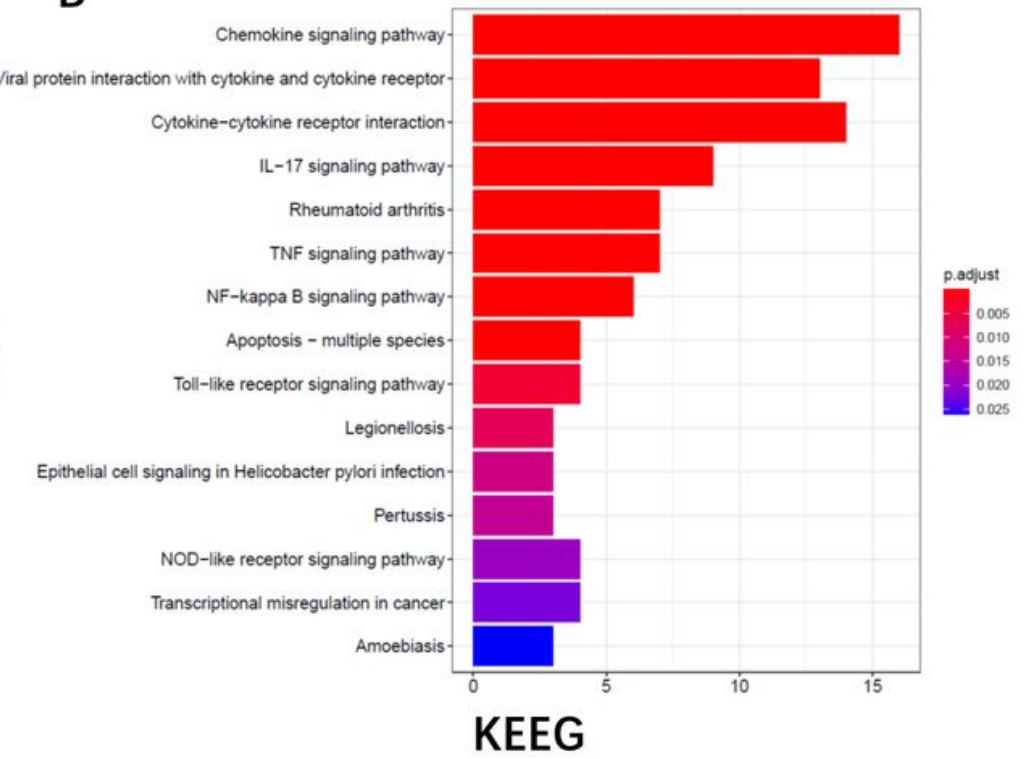

\section{Figure 6}

Functional enrichment analysis of the differentially expressed CXC Chemokines and their similar genes in patients with GBM using R software. A. Bar-plot of GO enrichment in biological process terms and molecular function terms. B. Bar-plot of KEGG enriched terms. 

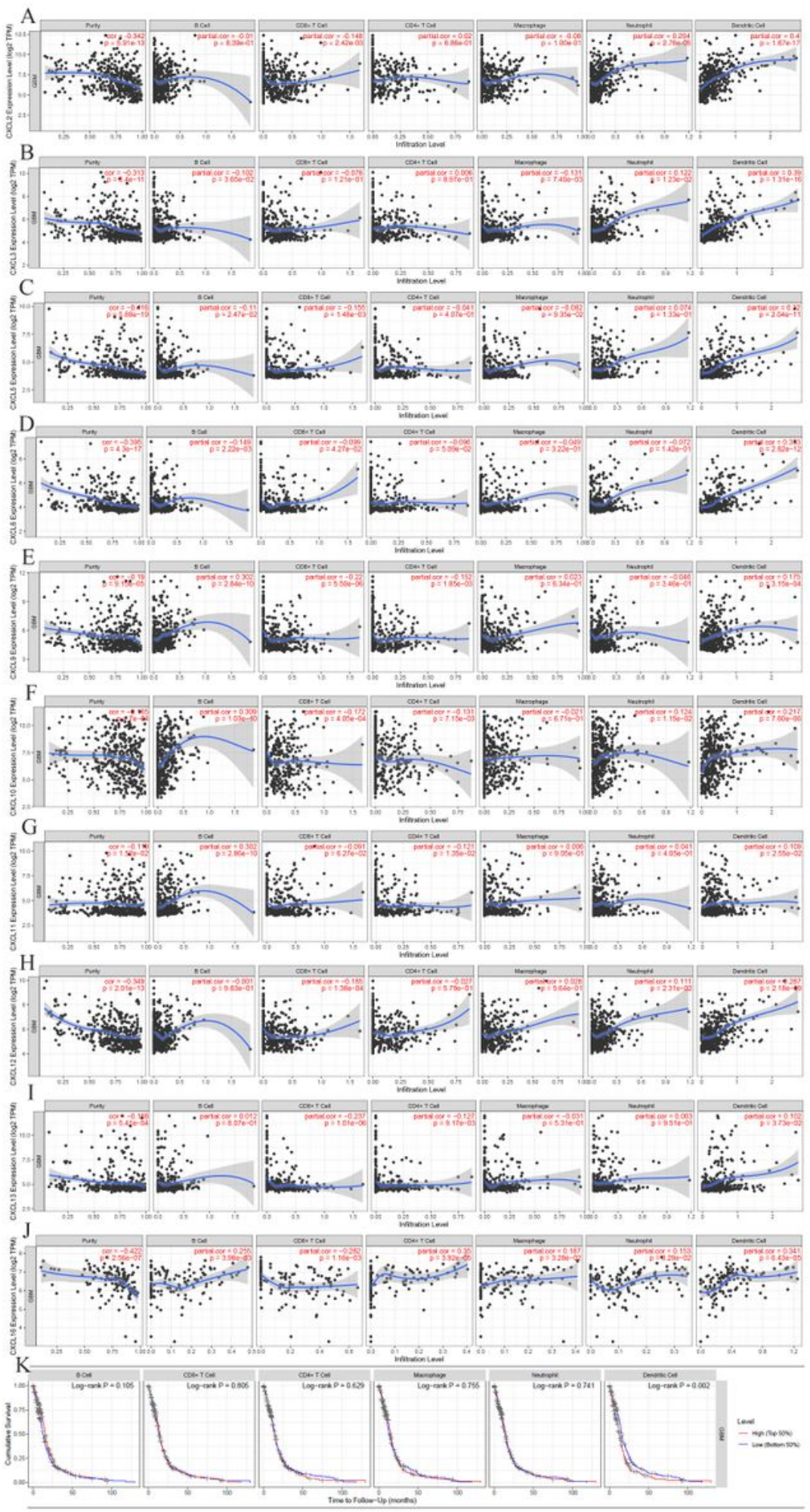

\section{Figure 7}

Immune cell infiltration correlated with expression of CXC chemokines and prognosis in GBM. A-J: Immune cell infiltration (B cells, CD4+ T cells, CD8+ T cells, Neutrophils, Macrophages, and Dendritic cells) of CXC chemokines in GBM. K: correlation of cumulative survival and immune cell infiltration in GBM using the Cox proportional hazard model. 